\title{
Factors Associated with Lifetime HIV Testing in Texas by Race/Ethnicity
}

\author{
Raquel A. Benavides-Torres ${ }^{1}$, Kristin M. Wall ${ }^{*}, 2$, Georgina Máyela Núñez Rocha ${ }^{3}$, \\ Dora Julia Onofre Rodríguez ${ }^{1}$ and Laura Hopson ${ }^{4}$
}

\author{
${ }^{I}$ Universidad Autónoma de Nuevo León, UANL. FAEN, CIDICS, Av. Universidad S/N Ciudad Universitaria San Nicolás \\ de los Garza Nuevo León, C.P. 66451, México \\ ${ }^{2}$ Department of Epidemiology, Rollins School of Public Health, Emory, University, Atlanta, GA, USA \\ ${ }^{3}$ Universidad Autónoma de Nuevo León, UANL, FaSPyN, Av. Eduardo Aguirre Pequeño y Yuriria, Col Mitras Centro, \\ Monterrey N. L. C. P. 64460, México \\ ${ }^{4}$ University at Albany, School of Social Welfare, New York, USA
}

\begin{abstract}
Introduction: In United States, roughly 1/5 of all HIV infected persons remain undiagnosed. Because HIV testing is critical to improve prevention efforts, more research is needed to understand the characteristics of individuals who get tested for HIV.

Methods: This secondary analysis of the 2010 Texas Behavioral Risk Factor Surveillance System used data from 9,744 respondents between 18-64 years of age to evaluate the relationship between demographic characteristics (gender, race/ethnicity, age, area of residence, education, marital status, employment status, and income), healthcare characteristics (insurance status, having a primary provider, and access to healthcare), and HIV risk behaviors with ever having received an HIV test.
\end{abstract}

Results: Significant associations between gender, age, area of residence, marital and employment status, and HIV risk behaviors and HIV testing in a Texas population by race/ethnicity were observed.

Conclusions: These findings have important implications for future research into racial/ethnic disparities between lifetime HIV testing, and can help guide practitioners who work with populations at risk for HIV/AIDS in Texas.

Keywords: Diagnosis, HIV, prevention, racial, screening.

\section{INTRODUCTION}

More than 1,100,000 people in the United States (US) are living with HIV/AIDS, and an estimated $20 \%$ of these infections remain undiagnosed $[1,2]$. As of 2008 , over 77,000 cases of HIV/AIDS have been reported in Texas, the 4th highest number of cumulative cases reported by state [3]. Knowledge of HIV status is critical for reducing the rates of HIV/AIDS transmission, and HIV testing has been an important part of HIV prevention efforts with improvements in test accuracy and availability [4]. In 2009, an estimated $45 \%$ of persons aged 18-64 reported ever having received an HIV test in the US [1].

Poor rates of routine testing and late diagnosis of HIV, which are common in the US, represent missed opportunities for linkage to care, treatment of HIV positive persons, and prevention of new infections. Of incident HIV diagnoses in 2008, one-third progressed to AIDS within 1 year, indicating these persons had likely been infected for the decade prior to diagnosis [1, 5]. In Texas, over one-third of all HIV diagnoses between 2003-2009 were late stage diagnoses, and

*Address correspondence to this author at the Department of Epidemiology, Rollins School of Public Health, Emory University, Atlanta, GA, USA; Tel: 512-785-4779; E-mail: kmwall@emory.edu an estimated one-third of Texans with known HIV diagnoses were not receiving care [6].

Various demographic, healthcare, and behavioral characteristics have been associated with ever having been tested for HIV including gender, age, and race. As of 2008, of those living with HIV/AIDS over the age of 13 in the US, roughly $21 \%$ of males and $18 \%$ of females had undiagnosed infections. By age, an estimated $58.9 \%$ of HIV positive persons aged 13-24 are living with undiagnosed HIV infections; this percentage is lower for other age groups [5].

HIV disproportionally affects blacks and Hispanics in the US relative to whites, with rates of infection nine and three times higher, respectively $(112.1 / 100,000$ for blacks and 40.5/100,000 for Hispanics versus 12.6/100,000 for whites) [1]. By race/ethnicity, undiagnosed HIV infection is more common among Asians/Pacific Islanders (26.0\%), and American Indians/Alaska Natives $(25.0 \%)$ relative to blacks/African Americans (21.4\%), whites (18.5\%), and Hispanic/Latinos (18.9\%) [5]. According to the CDC, over $30 \%$ of US men living with AIDS are black, and almost $20 \%$ are Hispanic. Among women living with AIDS, almost $60 \%$ are black and $20 \%$ are Hispanic. In addition, those who are tested late in the course of the disease are more likely to be black or Hispanic [7]. 
Whites have been reported to be less likely to receive an HIV test than blacks or Latinos [8]. The CDC estimates that $61.8 \%$ of blacks, $47.6 \%$ of Latinos, and $40.9 \%$ of whites have ever been tested for HIV. However, despite higher rates of ever testing among blacks and Hispanics, the disproportionately high rates of HIV/AIDS diagnoses among these subpopulations indicate that blacks and Hispanics would benefit from increased testing frequency to increase early diagnoses [1]. This is a particularly relevant issue in Texas due to its large Hispanic population. According to the 2010 US Census, over 9.4 million Hispanics live in Texas, representing $37.6 \%$ of the population [9]. Of AIDS diagnoses in Texas, $45 \%$ were among whites, $32 \%$ were among blacks, and 22\% were among Hispanics [3].

Other factors including area of residence, education, marital status, employment, income, and insurance status also influence rates of HIV testing. In a secondary analysis of 2005 and 2009 Behavioral Risk Factor Surveillance System (BRFSS) data, the relationship between metropolitan statistical area (MSA) and HIV testing was examined. Persons residing in urban areas were significantly more likely to have been ever tested for HIV relative to those residing in rural areas (43.6\% vs 32.2) [10]. Research also indicates that individuals who have not graduated from high school are less likely than those with higher levels of education to be tested, and those who are tested late in the course of the disease are likely to be less educated than those tested earlier [7, 11].

Findings from 2008 BRFSS data indicated that a relatively higher proportion of those never married/divorced/widowed/separated had ever been tested relative to those who were married/a member of an unmarried couple (40.2\% vs 36.8\%) [12]. The 2008 BRFSS data indicate that relatively equal proportions of those who were employed versus unemployed had ever been tested. A higher proportion of those making an annual household income $<\$ 15,000$ had ever been tested relative to those earning $\$ 15,000-\$ 50,000$ or over $\$ 50,000(43.2 \%$ versus $40.1 \%$ and $37.6 \%$, respectively) in the 2008 BRFSS [12].

Insurance status may affect HIV testing as insured individuals likely have more contact with health providers or health clinics and therefore more opportunities to get an HIV test. Data from the 2008 BRFSS indicated that a similar proportion of insured and uninsured had ever received an HIV test (37.5\% and $39.7 \%$, respectively) [12]. Barriers to HIV testing may represent unique challenges in Texas because of the large numbers of uninsured residents, which the US Census estimated to be $24.6 \%$ of the population for 2003-2005 [13].

An individual's relationship with a healthcare provider can also be an important factor in deciding whether to be tested for HIV. Physician endorsement of HIV testing is one of the most consistent predictors of HIV testing. Attitude toward the person providing the test is a factor that HIV test recipients have identified as important in their attitudes toward HIV testing [14]. In the 2008 BRFSS, a similar proportion of those with or without a primary healthcare provider had ever been tested $(37.8 \%$ and $38.7 \%)$ and a higher proportion of those having not seen a doctor in the past year because of cost had ever been tested for HIV (46.3\% and $36.4 \%)$ [12].
HIV risk behaviors may also be related to ever receiving an HIV test - roughly $28 \%$ of persons with any HIV risk factor had ever been tested using 2001-2009 NHIS data for those aged 18-64 [1]. A stronger predictor of HIV testing than actual behavior may be perception of risk for HIV, and research suggests that perceiving no risk for HIV is a barrier to testing [15].

During recent years, HIV transmission and progression prevention strategies have emerged, including early diagnosis of the infection. A delay in diagnosis until later stages may be associated with irreversible immune damage and related complications. Accordingly, the Joint United Nations Program on HIV/ADIS has developed a strategy to reduce infections in young people by half with a primary objective of increased access to HIV testing [16].

Given the high prevalence of HIV infections in Texas, many of which are undiagnosed, the relatively high rates of uninsured persons, and inconsistent research findings regarding HIV testing among Hispanics, more research is needed to understand how these and other factors influence HIV testing in Texas. This study investigated the association between demographic characteristics (gender, race/ethnicity, age, area of residence, education, marital status, employment status, and income), health care characteristics (insurance status, having a primary provider, and access to health care), and HIV risk behaviors with ever having received an HIV test using 2010 Texas BRFSS data.

\section{METHODS}

\section{Data Collection and Study Participants}

The BRFSS is a CDC supported state-based, random-digit telephone survey of the civilian, noninstitutionalized US population aged 18 years and older. Through a series of structured telephone interviews, states collect uniform data monthly on the behaviors and conditions that place adults at risk for chronic diseases, injuries, and preventable infectious diseases that are the leading causes of morbidity and mortality in the US. Information on BRFSS design and sampling methods are reported elsewhere [17].

This study was a secondary analysis of data obtained from the 2010 Texas BRFSS. Study participants were those who were asked to provide information regarding ever having been tested for HIV, which was only asked of respondents younger than 65 years of age.

\section{Measures}

The outcome of interest was ascertained with the following question: "Have you ever been tested for HIV? Do not count tests you may have had as part of a blood donation. Include testing fluid from your mouth." Responses to this question included 'yes', 'no', 'don't know/not sure', and 'refused to answer'.

Race/ethnicity was measured by combining an item that asked participants to identify their ethnicity as Hispanic or nonHispanic and another item that asked participants to identify their race. Response categories for the combined race/ethnicity variable are 'white (non-Hispanic)', 'black (non-Hispanic)', 'other (non-Hispanic)', 'multiracial (non-Hispanic)', 'Hispanic', 'don't know/not sure', and 'refused'. The 'other (non-Hispanic)' 
race category included Asian, Native Hawaiian or other Pacific Islander, and American Indian/Alaskan native.

Gender was measured with an item that directed interviewers to indicate the sex of the respondent and to ask the respondent's sex if necessary. Possible responses are 'male' and 'female'. Age was measured with the following question: "What is your age?" and responses were categorized as: '18 24 years', ' 25 - 34 years', ' 35 - 44 years', ' 45 - 54 years', and '55- 64 years'.

MSA was recorded as whether the participant was 'in the center city of MSA', 'outside the center city of a MSA but inside the county containing the center city', 'inside a suburban county of the MSA', 'in a MSA that has no city center', and 'not in a MSA'.

Education was measured with the following question: "What is the highest grade or year of school you completed?" Responses to this question were: 'Never attended school or only kindergarten', 'Grades 1 through 8 (Elementary)', 'Grades 9 through 11 (Some high school)', 'Grade 12 or GED (High school graduate)', 'College 1 year to 3 years (Some college or technical school)', 'College 4 years or more (College graduate)', and 'refused'.

Marital status was captured with the prompt "Are you..." and response options were 'married', 'divorced', 'widowed', 'separated', 'never married', 'member of an unmarried couple', 'don't know/not sure', and 'refused.'

Employment status was captured with the prompt: "Are you currently..." Responses included 'employed for wages', 'selfemployed', 'out of work for more than 1 year', 'out of work for less than 1 year', 'a homemaker', 'a student', 'retired', 'unable to work', and 'refused'.

Respondent income was captured with the question: "Is your annual household income from all sources..." Responses included 'less than 10,000 ', ' $\$ 10,000$ to less than $\$ 15,000$ ', ' $\$ 15,000$ to less than $\$ 20,000$ ', ' $\$ 20,000$ to less than $\$ 25,000$ ', ' $\$ 25,000$ to less than $\$ 35,000$ ', ' $\$ 35,000$ to less than $\$ 50,000$ ', ' $\$ 50,000$ to less than $\$ 75,000$ ', ' $\$ 75,000$ or more', 'don't know/not sure', and 'refused'.

Health care insurance was measured with the following question: "Do you have any kind of health care coverage, including health insurance, prepaid plans such as HMOs, or government plans such as Medicare?" Responses to this question are 'yes', 'no', 'don't know/not sure', and 'refused'.

A primary healthcare provider was measured with the following question: "Do you have one person you think of as your personal doctor or health care provider? (If "No" ask "Is there more than one or is there no person who you think of as your personal doctor or health care provider." Responses to this question are 'yes, only one', 'more than one', 'no', 'don't know/not sure', and 'refused'.

Healthcare access was measured by the question: "Was there a time in the past 12 months when you needed to see a doctor but could not because of cost?" Responses to this question are 'yes', 'no', 'don't know/not sure', and 'refused'.

HIV risk behavior was measured with the following question: "I am going to read you a list. When I am done, please tell me if any of the situations apply to you. You do not need to tell me which one. You have used intravenous drugs in the past year. You have been treated for a sexually transmitted or venereal disease in the past year. You have given or received money or drugs in exchange for sex in the past year. You had anal sex without a condom in the past year. Do any of these situations apply to you?" This question was only asked to respondents under the age of 65 . Responses to this question are 'yes', 'no', 'don't know/not sure', and 'refused'.

\section{Statistical Analyses}

Responses of 'don't know/not sure', or 'refused' were considered missing and excluded from the analysis, as were respondents with missing data for any covariate. All exposures of interest were categorical and tabulated as counts, percentages, and weighted percentages (weighted to account for differences in selection probabilities) stratified by the response to the HIV testing history question. Weighting was achieved using FINALWT to account for differences in selection probabilities. Differences between the distributions of weighted independent variables by HIV testing history were evaluated via $\chi^{2}$ tests using PROC SURVEYFREQ.

Crude odds ratios (ORs) and 95\% confidence intervals (CIs) were calculated to evaluate the association between weighted independent variables and HIV testing history using PROC SURVEYLOGISTIC. All exposure variables significant (Bonferroni corrected p-value $=0.0042$ ) in crude analyses were entered as predictors into a multivariate logistic regression model predictive of HIV testing history.

Effect measure modification by race/ethnicity was hypothesized a priori to effect the outcome of interest. Because effect measure modification by race/ethnicity was observed, separate multivariate logistic regression models were run for each race/ethnic group.

The potential for collinearity between exposure variables within each model was evaluated by cutoffs of condition indices $>30$ and variance decomposition proportions $>0.5$. Statistical analyses were performed with SAS v9.3 (Cary, North Carolina).

\section{RESULTS}

Of the 18,077 respondents in the 2010 Texas BRFSS, 11,518 were under the age of 65 and were asked whether they had ever received an HIV test, of which 9,744 had complete exposure information. Of those, 3,575 (36.69\%; weighted $39.99 \%$ ) had ever received an HIV test, 5,933 (60.89\%; weighted 57.62\%) had never received an HIV test, and $236(2.42 \%$; weighted $2.40 \%)$ responded 'don't know/not sure' or 'refused to answer'.

The final analysis was restricted to the 9,744 respondents with complete exposure information answering 'yes' or 'no' to have been ever tested for HIV. The 236 respondents answering 'don't know/not sure' or 'refused' were excluded from modeling analyses. These respondents were significantly $(\mathrm{p}<0.05)$ different from those that answered the HIV testing history question as either 'yes' or 'no' by race/ethnicity (more likely to be white or multiracial/other and less likely to be black or Hispanic), education (more likely to have attained a higher level of education), and marital status (more likely to be married and less likely to be separated or a member of an unmarried couple). 


\section{Respondent Demographics}

Respondents who had ever received an HIV test were significantly $(\mathrm{p}<0.05)$ more likely to be black (non-Hispanic), female, of a younger age category, live in the central city of a MSA, divorced, unable to work, be of a lower income bracket, have a primary healthcare provider, to have not accessed health care in the previous year due to cost, and to have at least one self-reported HIV risk behavior (Table 1).

\section{Multivariate Logistic Regression Models}

The multivariate logistic regression model included the following variables: gender, age, MSA, marital status, employment status, not seeking healthcare due to financial cost, and HIV risk behavior(s). Model results are shown stratified by race/ethnicity due to statistically significant effect measure modification by this variable. No collinearity was found. Models run within the stratum of the multiracial or other ethnic group did not have sufficient sample size, leading to perfect separation by several covariates (age, HIV risk, and marital status) and the inability to generate meaningful point estimates. Thus, only models for white (non-Hispanic), black (non-Hispanic), and Hispanic are presented.

Among white respondents, females were significantly more likely to have been ever tested for HIV relative to males $(\mathrm{aOR}=1.26 ; 95 \% \mathrm{CI}: 1.00-1.59)$. Respondents aged $25-34(\mathrm{aOR}=2.45 ; 95 \% \mathrm{CI}: 1.24-5.18)$ and $35-44(\mathrm{aOR}=$ 2.53; 95\%CI: $1.24-5.18$ ) were also significantly more likely to have been ever tested versus respondents' aged 18-24. White individuals residing outside the center city of a MSA $(\mathrm{aOR}=0.72 ; 95 \% \mathrm{CI}: 0.55-0.93)$ and not in a MSA $(\mathrm{aOR}=$ 0.52 ; $95 \% \mathrm{CI}: 0.39-0.70)$ were less likely to have tested relative to those residing in the center city of a MSA. Those who were divorced versus married $(\mathrm{aOR}=1.47 ; 95 \% \mathrm{CI}$ : 1.03-2.09), unable to work versus employed $(\mathrm{aOR}=2.15$; 95\% $\mathrm{CI}$ : 1.37-3.36), and reporting HIV risk behavior(s) (aOR $=2.51$; $95 \% \mathrm{CI}: 1.15-5.48)$ were also more likely to have ever tested for HIV (Table 2).

Among black (non-Hispanic) respondents, those not living in a MSA were significantly more likely to have been ever tested $(\mathrm{aOR}=3.29 ; 95 \% \mathrm{CI}: 1.11-9.78)$ relative to those living in the central city of a MSA. Black respondents who were widowed $(\mathrm{aOR}=0.33$; 95\%CI: $0.13-0.86)$ or separated $(\mathrm{aOR}=$ 0.14 ; $95 \%$ CI: $0.05-0.37$ ) were significantly less likely to have ever tested relative to married respondents (Table 2).

Among Hispanic respondents, females were significantly more likely to have been ever tested relative to males $(\mathrm{aOR}=$ 1.92; 95\%CI: 1.39-2.66). Additionally, those aged 25-35 versus those aged $18-24(\mathrm{aOR}=2.63 ; 95 \% \mathrm{CI}: 1.35-5.13)$ and divorced relative to married respondents $(\mathrm{aOR}=1.75$; 95\%CI: 1.06-2.90) were also more likely to have ever received an HIV test (Table 2).

\section{DISCUSSION}

These findings suggest that there are important relationships between gender, age, area of residence, marital and employment status, and HIV risk behaviors and HIV testing in a Texas population by race/ethnicity. Whites and Hispanics shared many significant predictors of ever receiving an HIV test that were not observed among blacks.
White and Hispanic women were significantly more likely to have been tested relative to men of the same racial/ethnic group, and whites and Hispanic in the 25-44 year age group were also significantly more like to have ever been tested for HIV relative to members of the same racial/ethnic group ages 18-24. Additionally, white and Hispanic respondents who were divorced versus married and those reporting at least one HIV risk behavior were more likely to be tested. Similarly, BRFSS data from four states found that $19 \%$ of respondents reported at least one risk behavior, which was different by gender (men 23\%; women 15\%), and that among this subgroup, younger age groups also had a higher likelihood of having received a recent HIV test; perception of risk also influenced HIV testing behaviors [18]. These findings are consistent with our study for white and Hispanic populations, and emphasize the need to further explore racial/ethnic differences in HIV testing behaviors. Another study using 2005 BRFSS data to evaluate testing rates and predictors of testing among racial/ethnic groups found that, similar to our study, gender was not a significant predictor among blacks; in contrast to our findings, this study showed that marital status was not a significant predictor among Hispanics [19].

MSA was a significant predictor among whites and blacks. Whites who lived outside the central city of a MSA and those who did not live in a MSA were significantly less likely to have been ever tested relative to those who lived in the central city of a MSA. In contrast, blacks not living in a MSA were more likely to have been ever tested relative to those residing in the central city of a MSA. Studies have shown that persons residing in rural areas are less likely to report ever receiving an HIV test, possibly contributing to relatively high rates of late-stage HIV diagnoses in rural areas [10]. Findings from this study indicate that area of residence needs to be further explored in relation to HIV testing by racial/ethnic groups with the goal of planning interventions and allocating resources targeted to high risk groups. The implications for these differences may be compounded by disparities in HIV diagnoses by race/ethnicity between urban and rural areas [20]. Additionally, employment status was a significant predictor among whites but not the other racial/ethnic groups - whites who were unable to work were more than twice as likely to have been ever tested for HIV relative to those who were employed for wages. Also, blacks that were widowed or separated were significantly less likely to have been tested relative to those who were married.

Overall, higher rates of testing were observed among blacks relative to other racial/ethnic groups. The higher rates of lifetime testing for blacks may be a result of increased perception of risk [21]. The lower rates of testing among Hispanics could be associated with a reluctance to discuss sexual issues in the Hispanic culture, a barrier to visiting clinics or medical centers for an HIV test, and possibly differential risk perception $[22,23]$. Additionally, the relationship between risk perceptions/behaviors and HIV testing may be more important for some ethnic groups than others. Fernandez et al., reported that Hispanic men who engaged in HIV risk behaviors were more likely to have been tested and to have been tested recently than men who 
Table 1. Respondent Demographics, Health Care Factors, and HIV Risk by HIV Testing History, 2010 Texas BRFSS

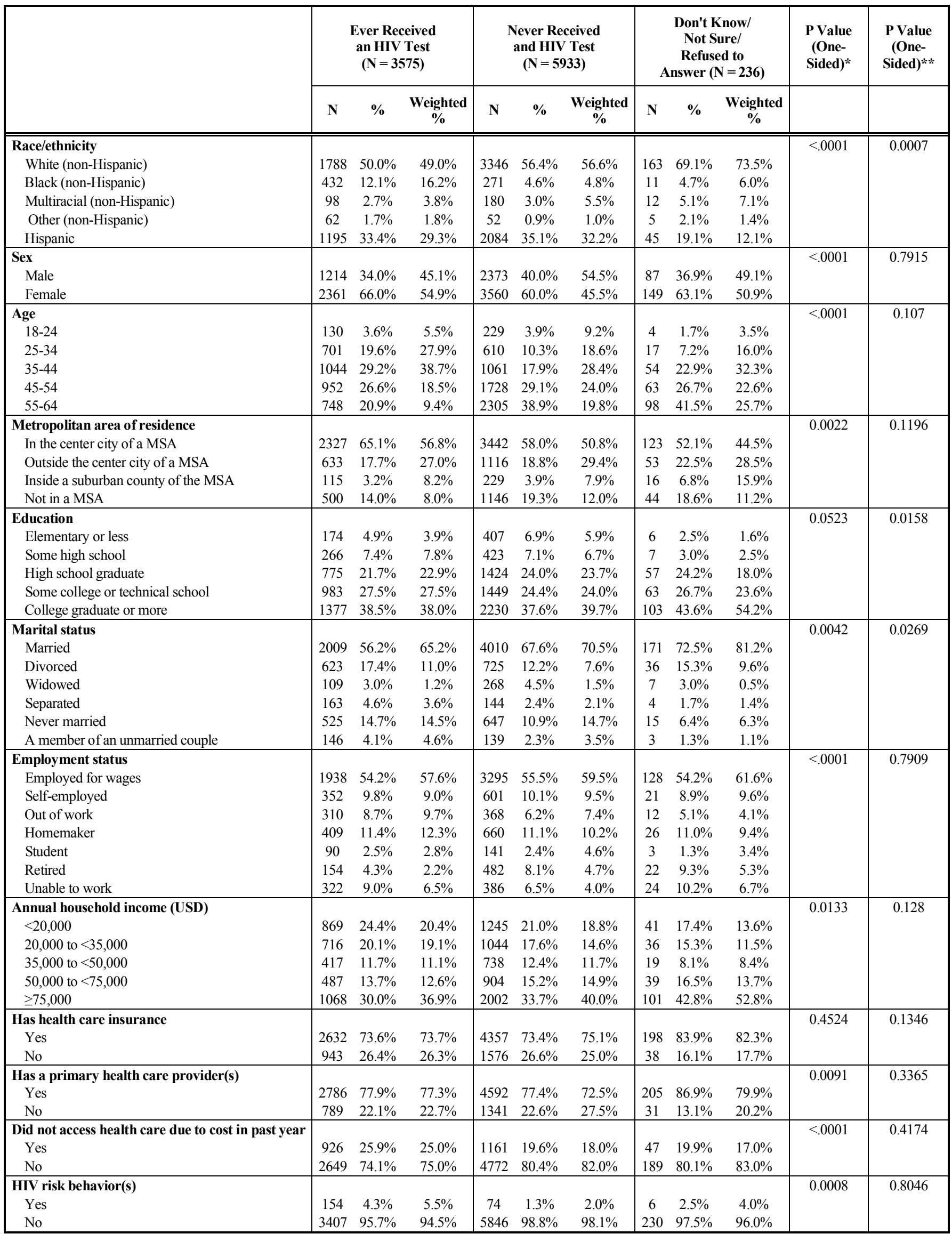

*Chi-square test for significant differences between those ever and never receiving an HIV test.

**Chi-square test for significant difference between those answering yes/no to ever having received an HIV test $v s$ non-responders/refusals. 
Table 2. Multivariate Logistic Regression Model of Factors Associated with Ever Receiving and HIV Test by Race/Ethnicity, 2010 Texas BRFSS

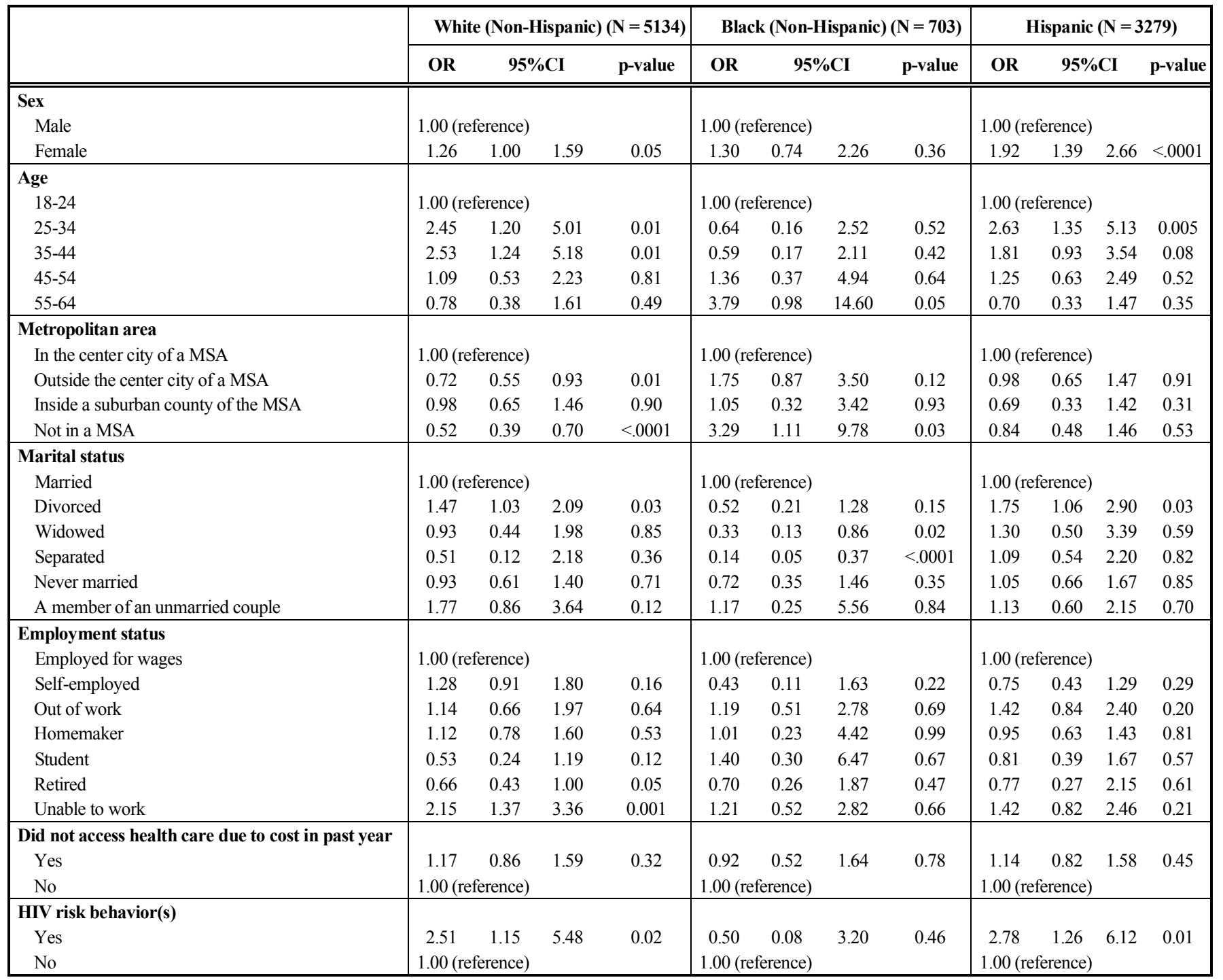

had not engaged in such behaviors [24]. Perceptions of risk may be a better predictor of HIV testing than actual riskbehavior, and a measurement of such perceptions may be a valuable addition to the BRFSS. Since perception of risk is cited as an important predictor of HIV testing, the CDC emphasizes the need for counselors involved in HIV prevention to assist clients in developing a more accurate perception of risk [25].

Limitations of the study include those inherent to the BRFSS, namely the cross-sectional nature of the data, selfreported data which may lead to information bias, potentially ambiguous question structures, and lack of inclusion of persons without telephones [17]. Further limitations to this analysis include the inability to evaluate the association between respondent knowledge of HIV and HIV treatments and HIV testing. Knowledge about treatments for HIV may be an important predictor since knowledge about treatments may reduce the fear of receiving a positive HIV test. Additional research should examine differences in knowledge about HIV among different racial/ethnic groups in order to develop strategies for improving differential knowledge and increasing rates of HIV testing for those most at risk. Studies reported that the level of awareness of HIV and HIV treatments varies by age, race, ethnicity, and education [26].

Our findings have implications for future studies and may aid healthcare professionals working with various racial/ethnic groups at-risk for HIV/AIDS in Texas. Because there were significant differences in the rates of HIV testing by demographic characteristics, more research is needed to investigate whether men and women or individuals from different ethnic groups need specialized interventions to promote HIV testing. Finding interventions that promote increased testing among Hispanics and blacks is especially important because of their increased risk for HIV/AIDS. Since the findings indicated that Hispanics were less likely to be tested than blacks, there is a critical need for more research studying the predictors of HIV testing for this population and interventions that may improve their rate of testing. 


\section{CONFLICT OF INTEREST}

No potential conflict of interest to disclose.

\section{ACKNOWLEDGEMENTS}

The authors are grateful to Elizabeth Abel, Ph.D. and Shirley Laffrey, Ph.D. at the University of Texas School of Nursing for their generous assistance and support.

\section{FUNDING SOURCES}

The paper was funded in part by a Pre-Doctoral Fellowship from the Universidad Autónoma de Nuevo León and PROMEP awarded to Raquel Benavides-Torres.

\section{REFERENCES}

[1] CDC. Vital signs: HIV testing and diagnosis among adults--United States, 2001-2009. MMWR Morb Mortal Wkly Rep 2010; 59(47): $1550-5$.

[2] Office of National AIDS Policy. National HIV/AIDS strategy. Available from: http://www.whitehou se.gov/administration/eop/ onap/nhas [cited 2011 December 2].

[3] CDC. HIV Surveillance Report, 2009. Available from: http://www.cdc.gov/hiv/topics/surveillance/resour ces/reports/ [cited 2011 December 3].

[4] Worthington C, Myers T. Desired elements of HIV testing services: test recipient perspectives. AIDS Patient Care STDS 2002; 16(11): $537-48$.

[5] CDC. HIV surveillance--United States, 1981-2008. MMWR Morb Mortal Wkly Rep 2011; 60(21): 689-93.

[6] Texas Department of State Health Services. HIVSTD Program. Available from: http://www.dshs.state. tx.us/hivstd/default.shtm [cited 2011 December 3].

[7] CDC. Late versus early testing of HIV--16 Sites, United States, 2000-2003. MMWR Morb Mortal Wkly Rep 2003; 52(25): 581-6.

[8] Ebrahim SH, Anderson JE, Weidle P, Purcell DW. Race/ethnic disparities in HIV testing and knowledge about treatment for HIV/AIDS: United States, 2001. AIDS Patient Care STDS 2004; 18(1): 27-33.

[9] United States Census Bureau. 2010 Census Interactive Popualtion Search. Available from: http://2010.cen sus.gov/2010census/ [cited 2011 December 3].

[10] Ohl ME, Perencevich E. Frequency of human immunodeficiency virus (HIV) testing in urban vs rural areas of the United States: results from a nationally-representative sample. BMC Public Health $2011 ; 11: 681$

[11] Johnson DF, Sorvillo FJ, Wohl AR, et al., Frequent failed early HIV detection in a high prevalence area: implications for prevention. AIDS Patient Care STDS 2003; 17(6): 277-82.
[12] Inungu J, Lewis A, Mustafa Y, Wood J, O'Brien S, Verdun D. HIV testing among adolescents and youth in the United States: update from the 2009 behavioral risk factor surveillance system. Open AIDS J 2011; 5: 80-5.

[13] United States Census Bureau. Income Climbs, Poverty Stabilizes, Uninsured Rate Increases. Available from: http://www.census.gov/ newsroom/releases/archives/income wealth/cb06-136.html [cited 2011 December 3].

[14] Kellerman SE, Lehman JS, Lansky A, et al. HIV testing within atrisk populations in the United States and the reasons for seeking or avoiding HIV testing. J Acquir Immune Defic Syndr 2002; 31(2): 202-10.

[15] Vermund SH, Wilson CM. Barriers to HIV testing--where next? Lancet 2002; 360(9341): 1186-7.

[16] Joint United Nations Program on HIV/AIDS. Strategy. Available from: http://www.unadis.org/en/media/unadis/contentassets/docum ents/unadispublication/2010/JC2034_UNADIS_Strategy_en.pdf [cited 2012 January 13].

[17] CDC. Behavioral Risk Factor Surveillance System, 2011. Office of Surveillance, Epidemiology, and Laboratory Services. Available from: http://www.cdc.gov/brfss/ [cited 2011 December 3].

[18] Takahashi TA, Johnson KM, Bradley KA. A population-based study of HIV testing practices and perceptions in 4 U.S. states. J Gen Intern Med 2005; 20(7): 618-22.

[19] Rountree MA, Chen L, Brown A, Pomeroy EC. HIV testing rates and testing locations, by race and ethnicity. Health Soc Work 2009; 34(4): 247-55

[20] CDC. HIV Surveillance in Urban and Nonurban Areas. Available from: http://www.cdc.gov/hiv/topics/surveillance/resources/slides/ urban-nonurban/index.htm [cited 2011 December 3].

[21] Inungu JN. Potential barriers to seeking human immunodeficiency virus testing among adults in the United States: data from the 1998 National Health Interview Survey. AIDS Patient Care STDS 2002; 16(6): 293-9.

[22] Marin BV. HIV prevention in the Hispanic community: sex, culture, and empowerment. J Transcult Nurs 2003; 14(3): 186-92.

[23] Lopez-Quintero C, Shtarkshall R, Neumark YD. Barriers to HIVtesting among Hispanics in the United States: analysis of the National Health Interview Survey, 2000. AIDS Patient Care STDS 2005; 19(10): 672-83.

[24] Fernández MI, Bowen GS, Perrino T. Promoting HIV testing among never-tested Hispanic men: a doctor's recommendation may suffice. AIDS Behav 2003; 7(3): 253-62.

[25] Fernández MI, Perrino T, Royal S, Ghany D, Bowen GS. To test or not to test: are Hispanic men at highest risk for HIV getting tested? AIDS Care 2002; 14(3): 375-84.

[26] CDC. Heterosexual transmission of HIV--29 states, 1999-2002. MMWR Morb Mortal Wkly Rep 2004; 53(6): 125-9. 